\title{
Health Status Unknown
}

National Cancer Institute

\section{Source}

National Cancer Institute. Health Status Unknown. NCI Thesaurus. Code C115222.

Indicates that a person's current status of health is not known. 